\title{
Componentes Individuales y Contextuales más Relevantes y su Relación con la Calidad de Vida Percibida por el Adulto Mayor del Cercado de Tacna. 2011.
}

\author{
Individual Components and Relevant Contexts with its Relation to \\ Quality of Life Perceived by the Elderly Tacna. 2011.
}

\author{
1 Wender Condori Chipana
}

\begin{abstract}
RESUMEN:
El presente estudio es de tipo descriptivo, transversal y correlacional, cuyo objetivo es determinar la relación entre los componentes individuales y contextuales con la calidad de vida percibida por el adulto mayor del cercado de Tacna. Población conformada por 97 adultos mayores. Se utilizó como instrumentos: cuestionario elaborado por el investigador para la valoración de los componentes individuales y contextuales, test de valoración simple de estado mental (SPSSQ) de Peiffer; escala de Katz, de Lawton y Brody para medir capacidad funcional, escala de Apoyo Familiar y la escala de la MGH para la valoración de la calidad de vida. Los resultados fueron, que la mayoría de los adultos mayores, están en el grupo etareo de 60 a 69 años de edad, con predominancia de sexo femenino, estado civil casado, procedencia de Tacna, con residencia de 20 años a más. Los componentes individuales como la salud fisica, el $48,5 \%$ refiere estar enfermo, el $45,4 \%$ en riesgo de enfermar, por presentar alguna sintomatología clínica, la salud psíquica, el $71,1 \%$ está en riesgo de presentar algún alteración cognitiva, el 20,6\% manifiestan presencia de enfermedad, según la valoración simple del estado mental el 77,3\% presenta función intelectual intacta, el $22,7 \%$ presenta de ligero a moderado deterioro cognitivo, el 59,8\% tiene baja integración social, el $51,5 \%$ refiere estar ni satisfecho, ni insatisfecho con su vida; respecto a la capacidad funcional el $61,9 \%$ es dependiente para las $A B V D$, el 90,7\% es dependiente para las AIVD, el 53,6\% refiere poca actividad en su tiempo libre. Según componentes contextuales el 41,2\% tienen primaria, el 59,8\% son de baja situación económica, el 49,5\% tiene bajo grado de apoyo social, según la calidad ambiental de su vivienda el $97,9 \%$ cuenta con servicios básicos y el 59,8\% esta satisfecho con su vivienda, el 47,4\% tiene poca accesibilidad a los servicios de salud y el 56,7\% manifiesta haber utilizado los servicios de salud en los últimos seis meses, el 40,2\% refiere haber utilizado los servicios del Ministerio de Saludy el $42,3 \%$ de los adultos mayores tienen muy baja calidad de vida. Concluimos que existe relación altamente significativa $(p>0,01)$ entre el nivel educativo y la calidad de vida, y relación significativa $(p>0,05)$ entre edad, sexo, estado civil, test de valoración simple de estado mental, capacidad funcional y actividad en su tiempo libre, y no existe relación entre procedencia, tiempo de residencia, salud fisica, psíquica, integración social, satisfacción con la vida, situación económica, apoyo social, calidad ambiental de su vivienda, accesibilidad y utilización de los servicios de salud. El mayor porcentaje de la población en estudio tiene de baja calidad de vida.
\end{abstract}

Palabras clave: calidad de vida, adulto mayor, componentes individuales y contextuales.

\section{ABSTRACT:}

This research study is a descriptive, cross-sectional and correlational, which aims to determine the relationship between individual and contextual components to quality of life perceived by the elderly of the center of Tacna. The study population consisted of 97 older adults. To collect data, we used the following instruments: questionnaire prepared by the investigator for the evaluation of individual components and contextual simple test of mental status evaluation (SPSSQ) of Peiffer, Katz scale of Lawton and Brody to measure capacity functional, Family Support Scale and the scale of the MGH for assessing the quality of life. The analyzed results show that older adults are among the 60 to 69 years old, predominantly female, married, Tacna origin, resident of 20 years more. Regarding the individual components as physical health, $48.5 \%$ reported being sick and $45.4 \%$ at risk of becoming ill they presented clinical symptoms, mental health, $71.1 \%$ are at risk of any cognitive impairment, $20.6 \%$ show the presence of disease, as assessed by simple mental status, $77.3 \%$ have intact intellectual function, $22.7 \%$ have mild to moderate cognitive impairment, $59.8 \%$ have low integration social, $51.5 \%$ reported being neither satisfied nor dissatisfied with his life regarding the functional capacity is dependent $61.9 \%$ for $A B V D, 90.7 \%$ is dependent for AIVD, $53.6 \%$ relates little activity in their leisure time. According to contextual components are $41.2 \%$ primary, $59.8 \%$ are of low economic status, $49.5 \%$ have low level of social support according to the environmental quality of your home $97.9 \%$ with basic services and $59.8 \%$ are satisfied with their housing, $47,4 \%$ have little access to health services and $56.7 \%$ report having used health services in the past six months, $40.2 \%$ reported have used the services of the Ministry of Health and $42.3 \%$ of older adults have very poor quality of life. We conclude that there is highly significant $(p>0.01)$ between educational level and quality of life and significant relationship ( $p>0.05)$ between age, sex, marital status, test simple mental status assessment, capacity functional activity in their free time, and no relation between source, length of residence, physical, mental, social integration, satisfaction with life, economic status, social support, environmental quality of housing, accessibility and utilization of services health. The highest percentage of the study group had lower quality of life. 


\section{I.-INTRODUCCIÓN.}

El siglo XX fue el período en que se ha producido la revolución de la longevidad y del envejecimiento. Muchos de nosotros hemos crecido en ese paradigma y algunos seguimos pensando que ese fenómeno se va a prolongar de manera indefinida.

En el siglo XXI, sin embargo, tendremos que enfrentar el desafio de hacer compatible la longevidad con la autonomía personal, para que el reto no sea el envejecimiento dependiente. Lo que tenemos que conseguir ahora no es que la gente viva más, sino que viva mejor y sin dependencia. Algunos investigadores ya proyectaban a finales del siglo pasado que para el inicio de este milenio la posibilidad de aumentar la expectativa de vida media en los países desarrollados era inexistente. $\mathrm{Y}$ de igual modo lo será en los países que ahora están iniciando su transición demográfica y la completarán en las próximas décadas como es el caso de Perú y muchos otros países de Latinoamérica. Lo que sí está ocurriendo es que la gente que llega a 60 años vive más años, aumentando el número de personas que alcanzan edades muy avanzadas de vida. Fenómeno denominado "envejecimiento del envejecimiento".

Junto a la transición demográfica hay otro fenómeno que se ha producido de manera concomitante: la transición epidemiológica, tomando protagonismo las enfermedades no transmisibles en detrimento de las transmisibles. Finalmente, y como consecuencia de estos dos fenómenos se ha producido un tercer hecho: la mortalidad se ha agrupado en los adultos mayores y en los muy mayores.

¿Qué consecuencias tienen estos cambios? Cuando uno se enfrenta con esta población que ya no va a vivir muchos años más, como hemos visto, la perspectiva de salud cambia. La principal prioridad de prolongar la vida deja de ser, para incidir en la "calidad de vida".

En el Perú, para el año 2005 se ha estimado que hubo 2 millones 146 mil adultos mayores que representan al $7,7 \%$ de la población del país. Este grupo poblacional tiene una tasa de crecimiento anual de $3.1 \%$. La dependencia de la población mayor se incrementaría con el tiempo de $13,27 \%$ el año 2025 e incrementarse a $34 \%$ el año 2050 .El aumento de este estrato poblacional se acompaña de modificaciones sustanciales en la estructura de la sociedad; por ejemplo desde el punto de vista ético social con relación a la Calidad de Vida, la población adulta mayor enfrenta una serie de problemas como escasa solidaridad, la individuación, el sentido de auto-trascendencia, las máximas potencialidades de experiencias vividas, la soledad, la marginación frente al joven, esta situación se enmarca en la sociedad postindustrial, en la que vivimos, de competencia, de globalización, en la cual priman los conceptos de individualismo, relativismo, subjetivismo y consumismo, generando una sensación de tedio hacia la vida, de tristeza; que genera inacción, repliega al ser sobre sí mismo, paraliza la acción y causa patologías, como la depresión y otros. Según el documento "Situación Actual de las Personas Adultas Mayores"(2002), del Ministerio de la Mujer y Desarrollo Social (MIMDES), la salud, constituye uno de los principales problemas de la población adulta mayor, asociado al aspecto socio-económico, son los elementos determinantes en la "calidad de vida" de este grupo poblacional.

El país no está preparado para enfrentar el envejecimiento poblacional ni sus consecuencias, por la mayor carga de enfermedades sobretodo de las no transmisibles que conducen a mayor utilización de los servicios de salud, mayor costo de la atención y mayor capacidad resolutiva de los establecimientos. La velocidad de crecimiento de la población adulta mayor y de las enfermedades no transmisibles supera cualquier capacidad financiera actual y será más grave sino se toman medidas desde ahora. La ciudad de Tacna no es ajena a esta realidad, que no se eximen de problemas de indole social y de salud tal como: como el desconocimiento del autocuidado de su salud, prevalencia de enfermedades crónico-degenerativas, abandono moral y económico, maltrato, desconocimiento de sus derechos, escasa integración, organización y participación en redes sociales afectando negativamente en la calidad de vida., por lo que es necesario e importante, proponer políticas sociales de intervención traducido en un plan gerontológico con el propósito de contribuir a un cambio en sus condiciones de vida, para mejorar la calidad de vida de las personas mayores y puedan alcanzar una vejez digna y satisfactoria, el presente trabajo de investigación pretende responder a la siguiente interrogante:

¿Cuáles son los componentes individuales y contextuales más relevantes, que se relacionan con la calidad de vida percibida por el adulto mayor del cercado de Tacna?

\section{Objetivos}

Determinar los componentes individuales y contextuales más relevantes y su relación con la calidad de vida percibida por el adulto mayor del cercado de Tacna. (Agosto a Diciembre del 2011)

Caracterizar los componentes individuales del adulto mayor según edad, sexo, estado civil, procedencia, tiempo de residencia, la salud física, psíquica, valoración simple del estado mental, integración social, satisfacción con la vida, capacidad funcional, actividad en su tiempo libre a través del cuestionario elaborado por el investigador.

Identificar los componentes contextuales según nivel educativo, situación económica, apoyo social, calidad ambiental de su vivienda, accesibilidad a los servicios de salud, utilización de los servicios de salud a través del cuestionario elaborado por el investigador.

Valorar el nivel de la calidad de vida utilizando el Cuestionario del Hospital General de Massachusett $(\mathrm{MGH})$.

\section{II.-MATERIALY MÉTODOS.}

Según la naturaleza del problema y los objetivos formulados por la investigación es de tipo descriptivo, prospectivo de corte transversal con un diseño de correlación. 


\section{Procedimiento.}

1) Selección del área de estudio: el presente estudio se realizó en los "Comités de adultos mayores de las Juntas Vecinales del cercado de Tacna", inscritos en el Programa del Adulto Mayor de la Municipalidad Provincial de Tacna. Se coordinó con la Gerencia de Desarrollo Social de la Municipalidad Provincial de Tacna, con la encargada del Programa del Adulto Mayor y con los Presidentes de la Juntas Vecinales seleccionadas. Los datos obtenidos, fueron ordenados y procesados con Software Statistica 17.0 (SPSS). Para el análisis de los datos obtenidos se consideraron los siguientes instrumentos estadísticos: distribución de frecuencias absolutas y porcentuales, prueba de $\mathrm{Ji}$ cuadrado (X2) y Correlación de Pearson.

Población de estudio: la población objetivo estuvo constituido por 678 adultos mayores de 60 años de edad correspondientes a 19 Juntas Vecinales adscritos a la Municipalidad Provincial de Tacna, registrados en el Programa del Adulto Mayor, que conforman el cercado de Tacna, para la muestra se utilizó la fórmula de poblaciones finitas y se procedió a la estratificación de la muestra.

Se utilizó como método: la encuesta, y técnica: la entrevista personal estructurada para la aplicación del instrumento.

La variable independiente constituido por los componentes individuales y contextuales del adulto mayor como: edad, sexo, estado civil, procedencia, tiempo de residencia, salud física, salud psíquica, integración social, capacidad funcional, actividad en su tiempo libre, nivel educativo, situación económica, apoyo social, calidad ambiental, accesibilidad a los servicios de salud, utilización de los servicios de salud; y la variable dependiente constituido por el nivel de la calidad de vida: calidad de vida muy baja, baja, media y alta.

\section{RESULTADOS.}

En la tabla $n^{\circ} 01$ se observa, que el $72,2 \%$ de los adultos mayores están comprendidos en el grupo etareo de 60 a 70 años de edad, el $61,9 \%$ son de sexo femenino, el $38,1 \%$ son casados, el $64,9 \%$ tiene un tiempo de residencia más de 20 años y el $46,4 \%$ manifestaron serprocedentes de Tacna.

En esta tabla $n^{\circ} 02$ se observa, el $48,5 \%$ de los adultos mayores presentan enfermedad y el $45,4 \%$ están riesgo de enfermar por presentar alguna sintomatología clínica relacionado a la salud fisica.

En relación a la variable salud psíquica, el 71,1\% están en riesgo de presentar algún problema cognitivo y el $20,6 \%$ manifiestan presentar problemas cognitivos (memoria).

En la valoración simple del estado mental, el 77,3\% presenta función intelectual intacta, el $14,4 \%$ presenta ligero deterioro cognitivo, el $59,8 \%$ tiene baja integración social y el $27,8 \%$ moderada integración, el $51,5 \%$ refiere estar ni satisfecho, ni insatisfecho con su vida.

En relación a la capacidad funcional el $61,9 \%$ es dependiente para realizar alguna de las actividades básicas de la vida diaria y el $38,1 \%$ es independiente, asimismo el $90,7 \%$ es dependiente para realizar alguna de las actividades instrumentales de la vida diaria y el $9,3 \%$ es independiente, en la variable actividad en su tiempo libre el $53,6 \%$ refiere ser poco activo y el $42,3 \%$ es activo.

En la tabla $n^{\circ} 03$ el presente cuadro se observa, que según nivel educativo el $41,2 \%$ de los adultos mayores tienen primaria, el $30,0 \%$ secundaria y el $15,5 \%$ sin instrucción, el 59,8\% son de situación económica baja y el $27,8 \%$ son de situación económica media, el 49,5\% tiene bajo grado de apoyo social y el $36,1 \%$ tiene moderado grado de apoyo social, según la variable calidad ambiental de su vivienda el $97,9 \%$ cuenta con servicios básicos y según satisfacción de su vivienda el $59,8 \%$ esta satisfecho, el $24,7 \%$ esta ni satisfecho y ni insatisfecho, el $47,4 \%$ refiere poca accesibilidad a los servicios de salud y el $56,7 \%$ refiere haber utilizado los servicios de salud en los últimos seis meses y el servicio más utilizado corresponde a los servicios del Ministerio de Salud con el 40,2\%.

En la tabla $n^{\circ} 04$ se observa, el $42,3 \%$ de los adultos mayores tienen un nivel de muy baja calidad de vida, seguido del $24,7 \%$ con baja calidad de vida, el $17,5 \%$ va de media a alta calidad de vida.

En la aplicación de Ji Cuadrado se determinó que existe relación significativa entre la edad, sexo, estado civil, test de valoración simple de estado mental, capacidad funcional ABVD y AIVD, actividad en su tiempo, y la calidad de vida percibida por el adulto mayor. Existe relación altamente significativa entre el nivel educativo y la calidad de vida percibida por el adulto mayor.

\section{DISCUSIÓN.}

El envejecimiento poblacional constituye el cambio demográfico más trascendental que enfrenta el mundo al inicio del nuevo milenio. Mientras que en el siglo pasado el problema principal de Salud Publica fue la supervivencia, en este siglo la mayor preocupación es la "calidad de vida". Las personas en estos días llegan a mayor edad y quieren envejecer con éxito. Este fenómeno es nuevo pero consecuentemente se asocia a otro fenómeno el "envejecimiento del envejecimiento". En donde cada vez más sujetos que llegan a más de 65 años en un muy buen estado de salud y desean mantenerlo, lo cual es comprensible porque la esperanza de vida se ha incrementado en algunas regiones del mundo. En el Perú, según el MIMDES la esperanza de vida es de 72 años para los hombres y 79 años para las mujeres.

Es por ello que se les debe de brindar las herramientas necesarias para que puedan desenvolverse con total autonomía, desmitificando ciertos mitos y estereotipos de que son eminentemente dependientes, de que no puedan realizar muchas de las actividades básicas de la vida diaria, considerado por muchos expertos como una seudopercepción. Según Moragas (1991) sostiene, que los adultos mayores cambian su personalidad según su entorno, siendo negativo cuando no les acoge y positivo cuando encuentran un ambiente propicio.

Por lo tanto en este contexto la familia juega un rol muy importante, no solo en el proceso regenerador de las 
enfermedades sino en su rehabilitación y acompañamiento (Citado por Coaguila Vera).

Kozier (1999) señala que la valoración integral del estado de salud del individuo es básica para la promoción de la salud. Conocedores de estos antecedentes, el presente estudio pretende determinar la relación entre los componentes individuales y contextuales y la calidad de vida percibida por el adulto mayor y de esta manera identificar los posibles problemas y riesgos que existen en esta población.

a).- En el presente estudio en relación a las características bio demográficas como la edad: el $72,2 \%$ de los adultos mayores están comprendidos en el grupo etareo de 60 a 70 años de edad. Estos resultados son similares con los encontrados por: Dueñas Gonzales el 51,1\%(2007), Coaguila Vera(2005), el 56,22\% de 60 a 69 años, Marshal Echevarría(2002), Joy Chion Li y cols(2006), el $67 \%$ de 60 a 70 años, Fernández Mamani ( 2006 ), el $68.5 \%$ de 60 a 69 años. Discrepando con los resultados de Inga Aranda -Vara Horna, que encontró en menor porcentaje el $32,8 \%$ de 60 a 69 años. Del total de la población el $61,9 \%$ de adultos mayores corresponde al sexo femenino y el $38,1 \%$ al sexo masculino. Las diferencias porcentuales son similares a los encontrados por Inga Aranda-Vara Horna (2000), el 52,5\% mujeres y el 47,5\% varones, Fernández Mamani ( 2006) el 55,6\% femenino y el $44,4 \%$ masculino, Durand Valencia (2004), el $70,40 \%$ femenino y el 29,60 masculino, Choquepuma Quispe (2006) el 65,43\% femenino y el $34,57 \%$ masculino, Joy Chion Li y cols (2006), el $51 \%$ femenino y el $49 \%$ masculino, Cornejo Cárdenas y cols (2006), el $55,3 \%$ femenino y el $44,7 \%$ masculino, Coaguila Vera (2005) el $56,22 \%$ y el $43,78 \%$ masculino, Leiton (2001) el $59.2 \%$ femenino y el $40,8 \%$ masculino OPS (1999), Marshal Echevarria (2002), MIMDES (2004), Dueñas Gonzales (2007), INEI(2007).

La OPS refiere que en la mayoría de los paises del mundo y Latinoamerica, la población femenina es mayor que la masculina, esto corrobora los datos encontrados por Staab (1999) (citado por Coaguila Vera 2005) (MIMDES 2005), refiere que a medida que envejece la población, las mujeres superan cada vez más el número de hombres, debido a la mayor supervivencia de las mujeres de $5 \mathrm{a} 8$ años más, que el varón, razón por la cual la tasa de viudez es mas alta en las ancianas. La causa de esta disparidad entre las tasas de mortalidad de hombres y mujeres es desconocida, pero se piensa que está relacionado con motivos biológicos (hormonales) y sociológicos. Por lo tanto estos cambios en el perfil epidemiológico nos invitan a tener mayor conocimiento en temas de salud de las mujeres y conocer las implicancias sociales que podrían derivar de una supervivencia mayor de las mujeres que de los hombres.

En relación al estado civil el $59,7 \%$ tienen pareja considerando a convivientes y casados, y el $45,3 \% \sin$ pareja considerando a viudos, solteros y divorciados sin pareja. Resultados que coinciden con lo informado por Papalia y Wendkos (1997) en Estados Unidos y Leiton y cols (2000) en Trujillo, (citados por Coaguila Vera
2005), Joy Chion $\mathrm{Li}$ (2006), el $76 \%$ son casados, viudos $18 \%$, separados $2 \%$, divorciados $1 \%$, solteros $3 \%$, Leiton y cols el $53 \%$ tienen unión estable, viudos $34,5 \%$, separados y divorciados $6,4 \%$ y el $6,1 \%$ solteros, Coaguila Vera (2005) el $68 \%$ casados, viudos $20 \%$,conivientes $6 \%$ solteros $4 \%$, divorciados $1,5 \%$, Durand Valencia (2004) el $43,10 \%$ casados, el $32,18 \%$ viudos, el $16,09 \%$ solteros, el $2,87 \%$ convivientes, el $5,75 \%$ divorciados, Choquepuma Quispe (2006), el 55.56 casado, $34,57 \%$ viudos, 6,17 divorciados, solteros $3,70 \%$, Inga Aranda-Vara Horna (2000), el $25 \%$ casados, 21,9 solteros, $6,3 \%$ convivientes, 43,8 viudos, $3,1 \%$ divorciados, INEI (2007), este último basado en el Censo Nacional de 2007 y en la Encuesta Nacional de Hogares (2006). Asimismo los datos obtenidos discrepan con Dueñas Gonzales (2007). Las diferencias en el estado civil tienen importantes implicancias económicas, sociales y relacionadas con la salud. Atchley (1992) indicaba que la mayoría de los hombres tienen un cónyuge con quien contar, pero muchas mujeres mayores tienen que buscar otras fuentes de apoyo social Saab (1996) (citado por Coaguila Vera 2005). Anzola señala que las investigaciones continúan mostrando que los casados viven mejor que los no casados en varias dimensiones económicas social, emocional, de cuidado durante el ciclo de vida, por tanto, en este grupo poblacional habría un buen apoyo.. Pero la viudez y la soledad constituyen un problema social, económico y psicológico de gran magnitud pues produce efectos negativos sobre el bienestar del adulto mayor, quien se ve vinculado a la pobreza, soledad, aislamiento, marginación.

Los grupos más vulnerables y de riesgo, según la OMS, son aquellos ancianos que viven solos en su vivienda, las mujeres ancianas viudas y los ancianos sin hijos corren el riesgo de sufrir deterioro en su calidad de vida OPS (1998) y Ruiperez y Llorente (1996), (citado por Durand Valencia 2004).

Referente a la procedencia, el $46,4 \%$ esta constituido por personas procedentes de Tacna, datos que concuerdan con el estudio de Durand Valencia en Arequipa (2004), el 64, 9\% son procedentes de Arequipa esto significa que el adulto mayor vive dentro o cerca de su entorno familiar y discrepamos con Inga Aranda - Vara Horna en Lima (2002) que la mayoria de los adultos mayores son migrantes de otros departamentos o provincias, asociado a la ausencia familiar lo que implica deterioro de la calidad de vida.

Según tiempo de residencia el $64,9 \%$ tiene más de 20 años de residencia en la ciudad, el $29,9 \%$ tiene 15 años y el $5,2 \%$ tiene entre 5 a 10 años de residencia.

Las características biodemográficas como: edad, sexo y el estado civil están relacionados significativamente con la calidad de vida.

b).- Loscomponentes individuales del adulto mayor: como la salud física, en donde el $48,5 \%$ de los adultos mayores refieren estar enfermo, y el $45,4 \%$ refieren estar en riesgo de enfermar, por presentar alguna sintomatología clínica. Es importante resaltar que el 
$81,11 \%$ de los adultos mayores refieren tener siempre o a veces "dolor", dato reportado por Coaguila Vera (2005). En la actualidad, es más fácil determinar la presencia o ausencia de una enfermedad que el nivel de salud.

Es evidente que cuando se ve afectada la salud fisica de la persona esto repercute en la autopercepción de su salud.

En relación a la variable la salud psíquica el resultado reporta, que $71,1 \%$ de la población en estudio manifiesta estar en riesgo en sus facultades mentales.

La capacidad de funcionalidad puede disminuir si algún trastorno, enfermedad crónica o lesión limita aptitudes físicas o mentales. En las personas adultas mayores, dicha reducción tiene consecuencias importantes para el trabajo, la jubilación, la salud y las necesidades de atención a largo plazo, así como el bienestar general (OPS/OMS-2000).

El estudio de los determinantes de la salud revela que hay una gama amplia de factores que contribuyen a mejorar la salud de la población de los adultos mayores o las pone en situación de riesgo. Estos factores raras veces existen por separado y por lo tanto es raro que respondan a soluciones unidimensionales, los factores de riesgo centrales son: el asilamiento social, la pobreza, la mal nutrición, la vida sedentaria, el estrés, la ansiedad y la depresión(OMS/OPS-2002).

En los factores de riesgo el individuo tiene un grado de control, la falta de ejercicio fisico es el potencialmente más modificable.

Las enfermedades crónicas pueden convertirse en una carga sanitaria y financiera, para la persona mayor, para la familia y para el sistema de atención de salud. Si estas enfermedades crónicas no se previenen o tratan adecuadamente, pueden conducir a una disminución considerable de la capacidad de funcionalidad del anciano y seguir siendo independiente. (OPS-1997).

Los resultados de la valoración simple del estado mental muestran, que el $77,3 \%$ de los adultos mayores presentan un estado mental intacto y el $22,7 \%$ con deterioro cognitivo de ligero a moderado y no se encontró ningún caso con deterioro severo. Resultados similares obtenidos por Coaguila Vera (2005), el $85,07 \%$ correspondiente a la función integra, $7,96 \%$ con ligero deterioro, moderado $4,98 \%$ y $1,99 \%$ deterioro severo.

Se entiende por deterioro cognitivo, la alteración de las facultades intelectuales de la persona entre los que destaca el deterioro de la orientación, de la memoria reciente, del razonamiento, del cálculo, del lenguaje, de la capacidad de realizar tareas complejas, de la capacidad de programación entre otras. Los trastornos cognitivos pueden deberse a diferentes causas desde trastornos ligeros como el "olvido benigno", hasta cuadros clínicos graves como la demencia (OPS-1999). Es probable que el funcionamiento cognitivo y afectivo en los adultos mayores decline durante una enfermedad o a causa de alguna lesión, como lo sostiene (Durante y Pedro 1998), que a pesar de que con la edad se produce una disminución de las funciones mentales, esto no parece sino al final de los 60 años y no es una disminución generalizada ya que un importante porcentaje de adultos no sufren declive alguno. Se considera que el organismo humano tiene capacidad de reserva que puede ser activada en la vejez y que incluso previene el declive. Existen estudios en donde estiman que entre el $5 \%$ y $10 \%$ de las personas de 65 años a más y el $20 \%$ al $30 \%$ de los adultos mayores de 85 años presentan demencia y la enfermedad del Alzheimer representa el $55 \%$ de ellas, según Tavares citado por Leiton y cols (2001).

En la variable integración social, el 59,8\% de los adultos mayores tiene baja integración y el $27,8 \%$ moderada integración social.

La integración social se entiende a los vínculos sociales que mantiene la persona adulta mayor. Depende de las redes de apoyo con la que cuente, entendiendo estas como aquellas relaciones significativas que una persona establece cotidianamente y a lo largo de su vida (Zapana 2001), especialmente con familias más cercanas, amigos, compañeros de trabajo, pareja entre otros. Se ha observado que tales redes juegan un papel vital en la adultez, convirtiéndose en el sustento emocional que permite en muchos casos sobrepasar las crisis, que se puedan presentar y además sean reconocidas como personas, mejorando su auto estima, su auto percepción, la eficacia personal y por ende la identidad (Peláez 2005).

Varios autores señalan, que la interacción con otros no solo en la adultez, sino a lo largo de toda la vida, se convierte en un factor protector frente a la aparición de patologías, trayendo como beneficio que la persona aumenta su capacidad cognitiva, tenga mejores habilidades de afrontamiento, module su afecto y aumente su calidad de vida (Cáceres 2004).

En relación a la variable satisfacción con la vida, que el $51,5 \%$ de los adultos mayores refieren estar ni satisfecho ni insatisfecho con su vida y el $28,9 \%$ esta satisfecho, datos que discrepan con Vara-Horna(2006), que el $80,3 \%$ están satisfechos Cornejo Cárdenas (2006), el 64\% satisfechos, Joy Chion Li (2006), 95\% satisfechos Durand Valencia (2004), 45\% satisfechos. La discrepancia está en que los estudios realizados en organizaciones de adultos mayores de diferentes instituciones, quienes refieren una buena integración social, reciben mayor refuerzo y atención social, los adultos mayores en su mayoría son jubilados.

La variable capacidad funcional es una de las mayores preocupaciones de las personas mayores es la pérdida de la autonomía en la realización de las actividades de la vida diaria. La capacidad funcional refleja la habilidad de los individuos para desarrollar actividades y papeles que son parte de la vida independiente y productiva.

Los niveles de independencia funcional se evaluaron considerando dos categorías:

Actividades básicas de la vida diaria (ABVD) como: bañarse, vestirse, ir al baño, comer, controlar esfinteres y subir y bajar gradas.

Actividades instrumentales de la vida diaria (AIVD), como: tareas del hogar, comprar, usar movilidad (cerca y 
lejos), manejar dinero y tomar medicina.

Cuyos resultados fueron: que el $61,9 \%$ son dependientes y el $38,1 \%$ son independientes a las actividades básicas $y$ el $90,7 \%$ son dependientes y el $9,3 \%$ son independientes. Pero los datos discrepan con Leiton Espinoza (2001), que refiere el $82 \%$ gozan de independencia en hombre y $76,4 \%$ en mujeres en realizar las ABVD y $68 \%$ en hombre y el $58,5 \%$ en mujeres en realizar las AIVD, Coaguila Vera (2005), reporta que el $85,57 \%$ son independientes $A B V D$ y el $65,1 \%$ en AIVD, Joy Chion Li (2006), reporta que el $87 \%$ de los encuestados son independientes para ambas actividades. Estas discrepancias pueden explicarse debido a que aunque el organismo experimenta un desgaste "sustancial", no significa déficit. El organismo funciona con reservas y la mayoría de los adultos mayores pueden tener una vida normal independientemente aunque con limitadas actividades de resistencia y de carga pesada, asimismo se sostiene que el envejecimiento no termina necesariamente en enfermedad y discapacidad. Si bien es cierto que los adultos mayores son capaces de tener una vida normal e independiente, hay que considerar que a medida que la gente envejece los niveles de reservas se reducen y las personas pueden ser menos capaces de cuidarse así mismos. También los procesos patológicos, estilo de vida, falta de entrenamiento, abandono de la actividad, estereotipos negativos, desconocimiento y sobreprotección, estarían atentando contra su independencia, su capacidad funcional y su calidad de vida. Esto explicaria los resultados obtenidos.

Al valorar la variable actividad en su tiempo libre, el $53,6 \%$ manifiesta ser poco activo a las actividades físicas y actividades pasivo-receptivas. Los datos encontrados difieren a los resultados de Joy Chion $\mathrm{Li}$, el $62 \%$ ocupa su tiempo libre en actividades fisicas o deportivas.

La actividad fisica en los adultos mayores constituye una necesidad biológica, disminuyendo el riesgo cardiovascular a la vez que incrementa la mayoría de las funciones fisiológicas, así como la actividad psicomotora, la capacidad para reaccionar ante una información exterior y modificar el movimiento y es también un positivo estimulador de las funciones psicológicas y la capacidad de los cognitivos, constituye un poderoso medio para aumentar el sentimiento de autoeficacia (Gonzales, 1995).

El ejercicio físico aumenta la fuerza o disminuye el riesgo de muerte, mejora el ánimo y reduce el impacto de otros factores de riesgo.

Existe relación significativa entre los componentes individuales como la valoración simple del estado mental, la capacidad funcional y el uso del tiempo libre con la calidad de vida.

c).- Los resultados de los componentes contextuales, según la variable nivel educativo, el $41,2 \%$ de los adultos mayores han cursado el nivel primario, el $30 \%$ el nivel secundario y el $15,5 \%$ sin instrucción.
Resultados similares a los encontrados por Leiton Espinoza (2001), el 47,2\% han cursado estudios de nivel primario y un $23 \%$ son analfabetos, Durand Valencia (2004), el $45 \%$ tienen primaria y el $17,8 \% \sin$ instrucción Coaguila Vera (2005), el 61,69\% tienen primaria y el $25,37 \%$ sin instrucción, Fernández Mamani (2006), el 40,7\% tienen primaria y el $37 \%$ son analfabetos, Vara Horna (2006), el 52,5\% tienen primaria y el $3,3 \%$ sin instrucción. Discrepamos con Joy Chion $\mathrm{Li}$ (2006), el 69\% tiene instrucción superior, $0 \%$ en el nivel de primaria y secundaria. El nivel educativo esta relacionado con el estado de salud. Es de esperar que las personas con altos niveles de educación vivan mas saludables (Staab, 1996).

Por lo tanto los niveles de instrucción encontrados en la población estudiada es de $56,7 \%$ con nivel primario o sin instrucción, lo que significa que están en riesgo de tener problemas de salud, ya que el alfabetismo y la educación contribuyen claramente al bienestar de los adultos mayores al permitir que se cumplan sus perspectivas económicas en una etapa temprana en la vida y que los adultos se preparen para la vejez.

En investigaciones previas se ha encontrado que las ancianas tienen menor nivel educativo que los ancianos (Leiton, 2001). El anciano va a tener menos probabilidades de presentar signos de enfermedad si su nivel educativo es alto.

En la variable situación económica esta relacionado con la salud, el $59,8 \%$ de los adultos mayores son de situación económica baja con ingresos de menos de 400 nuevos soles mensuales, datos similares a los encontrados por Leiton Espinoza (2001), el 60,1\% recibe entre 100 y 400 nuevos soles Durand Valencia (2004), el $51,44 \%$ son de situación económica mala y el $0,29 \%$ es buena, Herrera M. Soledad y Barros Carmen (2007), el $60 \%$ no tiene satisfechas sus condiciones económicas. Discrepamos con los resultados de Joy Chion $\mathrm{Li}$ (2006), el $80 \%$ de la población en estudio sus ingresos económicos son mayores a S/. 5,000 nuevos soles. Al analizar el grupo que no cuenta con un ingreso económico fijo o simplemente no lo tiene, es el grupo etareo femenino, por varios motivos: el hecho de ser mujer, por tener menos posibilidades de acceso al mercado laboral, por ser analfabeta. En estudios anteriores reflejan que los adultos mayores cuya situación económica es alta, tienen mejores perspectivas de llevar una vida más saludable.

En la valoración de apoyo social los resultados muestran, el $49,5 \%$ de los adultos mayores tienen un bajo grado de apoyo socio familiar, datos similares a los obtenidos por Durand Valencia (2004), el $21,84 \%$ no tienen apoyo social nunca y el $31,61 \%$ refiere a veces datos que discrepan con Leiton Espinoza (2001), el 44\% viven con familias extensas, Vara Horna (2000), el $80,6, \%$ de mujeres y $59,3 \%$ de varones se sienten felices como amigos, el $67,7 \%$ de mujeres y $55,6 \%$ de varones se sienten abiertos y receptivos frente a las amistades. Entonces este grupo es de mucho cuidado, puede repercutir en su estado cognitivo y afectivo

El apoyo social se define en términos de transacciones interpersonales, que implican ayuda, afecto y 
afirmación (Guzmán y otros 2003).

Shunker y Brownel (1984), definen apoyo social como intercambio de recursos entre al menos dos individuos el proveedor y el receptor. Mac Dowell y Newell (1996) a diferencia de los autores anteriores, destacan que lo que importa es más bien la "disponibilidad" de personas en quien el sujeto pueda confiar o en quien pueda descansar y que lo que hacen sentirse cuidado, valorado como persona (citado por Leiton 2001)

Cuando se habla de apoyo la atención se pone en quien lo recibe, pero usualmente se trate de un intercambio en que recibe y se otorga. Cuando el intercambio es reciproco se generan efectos positivos para los participantes. Cuando se da más de lo que se recibe se experimenta frustración y sobrecarga, cuando se recibe más podría sentir dependencia y endeudamiento (Guzmán 2003). Conviene establecer que las relaciones en sus formas externas, pueden ser apoyadores o positivos de afecto, cercanía y cooperación o pueden ser disturbadoras o negativas de desafecto, de hostilidad, de lejanía y entorpecimiento (Rook 1984) citado por Herrera My Barros C. (2008).

En la variable calidad ambiental de su vivienda se evaluaron dos aspectos: servicios básicos y satisfacción con su vivienda. Los resultados obtenidos fueron: el $97,9 \%$ de las viviendas de los adultos mayores cuenta con servicios básicos y asimismo el $59,8 \%$ esta satisfecho con su vivienda. Datos similares encontrados por Joy Chion Li (2006), el 100\% posee casa propia y vive con sus familiares. Por consiguiente los adultos mayores en estudio son propietarios y satisfechos de su vivienda porque todavía viven con su familia. Tal parece que la familia sigue siendo la principal fuente de apoyo emocional, seguridad, cuidado, afecto, ya que constituye un mecanismo natural de atención y es la opción preferida por los ancianos. Al analizar los resultados podemos sostener que una alta proporción de ancianos viven con su familia y a la vez manifestaron estar satisfechos de su situación familiar(OPS 1999).

En relación a la accesibilidad a los servicios de salud y la utilización de los servicios de salud, el 47,4\% de los adultos mayores refieren, que tienen poca accesibilidad al servicio y el $56,7 \%$ manifestó haber utilizado los servicios de salud por lo menos una vez en los últimos seis meses, por presentar dolencias y ha sido al sistema de servicios del Ministerio de Salud, datos similares obtenido por Villanueva B. Maritza (2009), que las mujeres tienen mayor acceso a los servicios socio sanitarios, el $68,2 \%$ asisten a consulta médica, de los cuales el $57 \%$ están afiliados al Sistema Integral de Salud en la ciudad de Iquitos y los datos son similares en las otras ciudades de estudio. Los resultados discrepan con (Leiton 2001), que sólo el $20 \%$ de los adultos mayores acudió a un servicio de salud. Al analizar el uso de los servicios de salud el grupo que asistió ha sido por presentar una enfermedad aguda. Los resultados muestran que el $33 \%$ no acudió a un servicio de salud formal. Si consideramos la vulnerabilidad y el riesgo a incrementar la severidad de los daños en este grupo poblacional, es preocupante contar con una demanda no efectiva. Una de las grandes barreras sería que no cuentan con un seguro de salud, podría estar explicando en parte la poca accesibilidad y el no uso.

Existe relación altamente significativa entre el nivel educativo y la calidad de vida

Existe relación significativa entre la satisfacción con su vivienda y la calidad de vida percibida por el adulto mayor.

d).- Según nivel de calidad de vida, el $42,3 \%$ de los adultos mayores tienen muy bajo nivel de calidad de vida y el $24,7 \%$ tiene bajo nivel calidad de vida, resultados similares hallados por (Marshal Echevarria 2006), cuyos resultados fueron el $47,7 \%$ tiene bajo nivel de calidad de vida, el $23,8 \%$ tiene nivel medio de calidad de vida, el $18,5 \%$ tiene muy bajo nivel de calidad de vida y el $10 \%$ tiene un alto nivel de calidad de vida respectivamente, (Choquepuma Quispe 2006), obtuvo que el $58,02 \%$ tiene mala calidad de vida y el $41,98 \%$ buena calidad de vida, (Durand Valencia 2004), que el $52,87 \%$ regular calidad de vida y el $8,33 \%$ mala calidad de vida,(Dueñas Gonzales 2007). En investigaciones realizadas en Guantánamo por Fernández Martínez y en la Ciudad de La Habana por López Fernández encuentran predominio de calidad de vida baja 1998 (citado por Dueñas Gonzales 2007).Discrepamos con Joy Chion Li. De este hecho inferimos, que la calidad de vida esta dado por las características particulares en el aspecto social, cultural y económico en las diferentes poblaciones de estudio.

$\mathrm{Si}$ consideramos tanto los niveles muy bajo y bajo indican, el $66,0 \%$ tienen un bajo nivel de calidad de vida, lo que indudablemente repercute en las dimensiones fisicas, psicológicas y sociales del adulto mayor deteriorando su calidad de vida.

Estos resultados pudieran estar ligados a la crisis socioeconómica, que implican exclusión y marginación, asociado a la escasa presencia de programas y proyectos socio sanitario por parte del Estado.

\section{CONCLUSIONES.}

El $72,2 \%$ de los adultos mayores están comprendidos en el grupo etareo de 60 a 69 años de edad, el $61,9 \%$ son de sexo femenino, el $59,7 \%$ tienen pareja(casados y convivientes) y el $46,3 \%$ no tiene pareja(solteros, viudos), el $64,9 \%$ tiene tiempo de residencia de 20 años a más y el 46,4\% manifestaron ser procedentes de Tacna.

Los componentes individuales de mayor predominancia estadística en los adultos mayores en estudio son: el 48,5\% presentan enfermedad aguda o crónica y el $45,4 \%$ están en riesgo de enfermar, por presentar sintomatología clínica.

En relación a la variable salud psíquica, el $71,1 \%$ está en riesgo de presentar algún problema en sus facultades mentales y el 20,6\% manifiestan presentar problemas cognitivos esporádicos. En la valoración simple del 
estado mental, el 77,3\% presenta función intelectual intacta, el 14,4\% presenta ligero deterioro cognitivo, el $59,8 \%$ tiene baja integración social y el $27,8 \%$ moderada integración, el $51,5 \%$ refiere estar ni satisfecho, ni insatisfecho con su vida. En relación a la capacidad funcional el $61,9 \%$ es dependiente para realizar alguna de las actividades básicas de la vida diaria y el $90,7 \%$ es dependiente para realizar alguna de las actividades instrumentales de la vida diaria, en la variable actividad en su tiempo libre el 53,6\% refiere ser poco activo.

Según los componentes contextuales el $41,2 \%$ de los adultos mayores tienen nivel primario, el $30,0 \%$ secundaria y el $15,5 \%$ sin instrucción, el $59,8 \%$ son de situación económica baja y el $27,8 \%$ son de situación económica media, el $49,5 \%$ tiene bajo grado de apoyo social, y el $36,1 \%$ tiene moderado grado de apoyo social , según la variable calidad ambiental de su vivienda el $97,9 \%$ cuenta con servicios básicos y el $59,8 \%$ está satisfecho con su vivienda, el $24,7 \%$ refiere estar ni satisfecho y ni insatisfecho, el $47,4 \%$ manifiesta poca accesibilidad a los servicios de salud, y el $56,7 \%$ refiere haber utilizado los servicios de salud en los últimos seis meses y el servicio más utilizado corresponde al Ministerio de Salud con el 40,2\%.

Según el nivel de calidad de vida, el 66,0\% de los adultos mayores tienen un bajo nivel de calidad de vida percibida y el $17,5 \%$ tienen un nivel de media a alta calidad de vida.

Los adultos mayores de la población en estudio presentaron un nivel de muy baja calidad de vida percibida.

Los componentes individuales como la edad, sexo, estado civil, valoración simple de estado mental, la capacidad funcional (actividades básicas e instrumentales de la vida diaria) y actividad que realiza en su tiempo libre, se relacionan con el nivel de la calidad de vida percibida por los adultos mayores siendo estadísticamente significativo.

Asimismo los componentes contextuales como el nivel educativo y la satisfacción con su vivienda, se relacionan con el nivel de la calidad de vida percibida siendo el primero altamente significativo y el segundo significativo.

\section{REFERENCIAS BIBLIOGRÁFICAS.}

1.- Anzola Pérez E. "La Atención de los Ancianos: Un desafio para los años noventa. OPS- Washington E.U.A.1994

2.- Aspectos conceptuales y metodológicos en la ve jez. 2000 , Dirección URL: http://www.geocites.com//psicología/VEJEZ.rtf>

3.- Carmen Barros; Forthes Alicia; Avendaño Cecilia. "Componentes de la calidad de vida del adulto mayor y factores asociados". Informe Técnico Fondecy 1020643. Santiago de Chile 2004
4.- Cancha Condori, M. "Maltrato familiar percibido por el anciano residente en la Asociación de Vivienda San Francisco-Gregorio Albarracín". Tesis para optar titulo de Licenciada en Enfermería.

5.- Centro Latinoamericano y Caribeño de Demografia (CELADE). "Indicadores de calidad de vida en la vejez". Santiago de Chile, Publicaciones de las Naciones Unidas.2006

6.- Coaguila Vera Elvira, "Valoración integral del adulto mayor y funcionamiento familiar Centro de Salud Víctor Raúl Hinojosa Simón Bolívar. Tesis para optar grado de Doctor en Enfermeria.Arequipa.2005

7.- Cornejo Cárdenas L e Icaza Palma P. "Satisfacción con la vida en adultos mayores "Tesis para optar grado de Licenciada en Psicología. Programa Profesional de Psicología. Universidad Católica Santa María. Arequipa .2006

8.- Chiavenato, Idalberto. "Introducción a la Teoría General de la Administración" Mc Graw-Hill Interamericana. Editores, S.A de C. V. México. 2000

9.- Choquepuma Quispe Y, Quispe Tacca F, "Funcionamiento familiar y calidad de vida del adulto mayor del pueblo joven Alto de la Libertad" Tesis optar titulo de Profesional en Enfermería. UNSA. Arequipa.2005.

10.- Dueñas Gonzales Dianelis M, "Calidad de vida percibida en adultos mayores de la provincia de Matanzas". Cuba 2007.

11.- Durand Valencia R y Pérez Apaza A, "Calidad de vida del adulto mayor en el Centro del Adulto Mayor Essalud, Asilo Lira, Francisco Bolognesi y Chochowasi Hunter. Tesis para optar titulo de Profesional de Enfermería.UNSA.Arequipa.2004.

12.- Encuesta Nacional de Hogares del Perú. 2006

13.- Fernández Ballesteros, R. (1992) "Calidad de vida en la vejez”. Investigación. España.1992

14.- Fernández Ballesteros R. Zamarrón, M. y Macía, A. "Calidad de Vida en la Vejez en los distintos contextos". Ministerio de Trabajo y Asuntos Sociales. Instituto Nacional de Servicios Sociales (Inmerso). Madrid, España.1997.

15.- Fernández Mamani E.'Influencia de la actitud del adulto mayor en su autocuidado" Tesis para optar el titulo de Licenciada en Enfermeria UNJBG.Tacna.2006

16.- Galarza Escarrillo Y." Proceso de evaluación de la calidad de vida" Universidad Autónoma de Mexico, 2007

17.- Galinski Hernández, Z. "Elaboración un Índice de 
5.- Centro Latinoamericano y Caribeño de Demografia (CELADE). "Indicadores de calidad de vida en la vejez". Santiago de Chile, Publicaciones de las Naciones Unidas. 2006

6.- Coaguila Vera Elvira, "Valoración integral del adulto mayor y funcionamiento familiar Centro de Salud Víctor Raúl Hinojosa Simón Bolívar. Tesis para optar grado de Doctor en Enfermeria.Arequipa.2005

7.- Cornejo Cárdenas L e Icaza Palma P. "Satisfacción con la vida en adultos mayores "Tesis para optar grado de Licenciada en Psicología. Programa Profesional de Psicología. Universidad Católica Santa Maria. Arequipa .2006

8.- Chiavenato, Idalberto. "Introducción a la Teoría General de la Administración" Mc Graw-Hill Interamericana. Editores, S.A de C. V. México. 2000

9.- Choquepuma Quispe Y, Quispe Tacca F,

23.- Maldonado Guzmán G y Mendiola Infante S. "Auto percepción de la calidad de vida de los adultos mayores" México .2004.

24.- Marriner Toney, Ann, Modelos y Teorias de Enfermería. Harcourt Brace. Madrid España.2007

25.- Maya, L. H Componentes de la calidad de vida en adultos mayores. Colombia. 1997 En http:www.funibre.org/documento/lamaya.hotml.

26.- Manfred Max-Neff. "Desarrollo a Escala Humana". Una opción para el futuro. CEPAUR.Colombia.2000

27.- Ministerio de Promoción de la Mujer y del Desarrollo Humano. Plan Nacional para las Personas adultas mayores 2006-2012.

28.- Morales MF, Salas A. Sánchez. MA .Editores. La atención de los ancianos. Un desafio para los años noventa. Publicación Científica No. 146 Washington OPS/OMS. 1990 www.facmed.unam.mx/deptos/salud/censena nza/spivsa/anciano/cinco.pdf

29.- Moragas R. Gerontología Social. Envejecimiento y calidad de vida.

Barcelona. Editorial Herder.2004

30.- Montenegro, O.'Reflexiones sobre envejecimiento y calidad de vida". Investigación. Universidad de Alicante. España. 1992.
31.- Leiton Espinoza y Cols. Perfil del adulto mayor en la provincia de Trujillo Universidad Nacional de Trujillo y el Instituto para el desarrollo humano y bienestar social. 2000 .

32.- Leturia, J y Col..." La Valoración de las Personas Mayores" lera Edición, Editorial Caritas Española. España. 2001.

33.- OMS. Programa sobre envejecimiento y salud. Ginebra 1998.

34.- OPS. "Envejecimiento activo, un marco político, documento presentado a la Segunda Asamblea Mundial de la Naciones Unidas sobre envejecimiento". Madrid. España. 2002

35.- Orfelio, G y Cols."Diseño de Investigaciones" Segunda Edición. Editorial Mac Graw-Hill/ Interamericana. España.1999.

36.- Peña Pérez B, Terán Trillo M, Moreno Aguilera F y Bazán Castro M, "Auto percepción de la calidad de vida del adulto mayor en la Clínica de Medicina Familiar Oriente del ISSSTE. México. Revista Especializada en Medico Quirúrgico México. $2009,14(2) 53-61$

37.-Pontificia Universidad Católica de Chile, "Encuesta Nacional sobre calidad de vida en la vejez en Chile".2008. Reyes, T."Salud en la Tercera Edad y Calidad de vida. Revista Cubana Vol. IV $\mathrm{N}^{\circ} 1$ Cuba.2002

38.- Ruiperez, I. "II Asamblea Mundial sobre el Envejecimiento" Revista de G e riatría y Gerontología. España.2002.

39.- Schalock R y Verdugo M.A. Calidad de vida: Manual para profesionales de la educación, salud, y servicios sociales. Madrid. Editorial Alianza.2003

40.- Villanueva B Maritza y cols "Factores predictores de la Calidad de vida de personas mayores de Iquitos". Universidad Nacional de la Amazonia Peruana. 2009.

41.- Yanguas Lezaun J.L. "Análisis de la calidad de vida relacionado con la salud en la vejez desde una perspectiva multidimensional". Madrid. Ministerio de Trabajo y Asuntos Sociales.2006.

\section{Correspondencia:}

Wender Florencio Condori Chipana Ciudad Universitaria Fundo "Los Granados"

Av. Miraflores s/n. Tacna. Perú. condoriwender@hotmail.com 